\title{
Structural basis for bisphosphonate-mediated inhibition of Leishmania major FPPS
}

\author{
P Azurmendi ${ }^{1}$, S Maheshwari ${ }^{2}$, W Hong ${ }^{2}$, M Murphy ${ }^{3}$, E Oldfield ${ }^{4}$, L Amzel ${ }^{5}$, M Silva ${ }^{6}$, S Gabelli $^{7}$ \\ ${ }^{1}$ Department of Biophysics and Biophysical Chemistry, Baltimore, MD, ${ }^{2}$ Department of Biophysics \\ and Biophysical Chemistry, Johns Hopkins University School of Medicine, Baltimore, MD, ${ }^{3}$ Cytiva, \\ Marlborough, MA, ${ }^{4}$ Department of Chemistry, University of Illinois at Urbana-Champaign, Urbana, \\ IL, ${ }^{5}$ Johns Hopkins University School of Medicine, Baltimore, MD, ${ }^{6}$ Global Health and Tropical \\ Medicine, Institute of Hygiene and Tropical Medicine, Lisbon, Portugal, ${ }^{7}$ Johns Hopkins \\ University, Ellicott City, MD \\ pazurme1@jhu.edu
}

Leishmaniasis, a major parasitic disease caused by infection with parasites of the genus Leishmania, affects about 12 million people in 98 countries. The most common form of leishmaniasis, cutaneous leishmaniasis, is caused by Leishmania major. Nitrogen containing bisphosphonates have been shown to have antiparasitic activity against Leishmania in vitro, by targeting the parasite farnesyl diphosphate synthase (FPPS), an enzyme essential for the promastigote and amastigote stages of Leishmania major. Bisphosphonates represent a compelling alternative for the treatment for leishmaniasis due to their safety in humans compared to current drug treatments, which have limitations because of high toxicity and drug resistance. The X-ray crystallographic structures of complexes of LmFPPS with three bisphosphonate inhibitors and $\mathrm{Ca} 2+$ at 2.25, 2.30 and $1.55 \AA$ resolution provided key information about the interaction with the inhibitors and the protein. The complex of LmFPPS with hydrogen (2-(1hexyl-1H-imidazol-3-ium-3-yl)-1-phosphonoethyl) phosphonate (1216) displays well resolved electron density for the bisphosphonate as well as for the substrate isopentenyl pyrophosphate (IPP) and three Ca2+ ions. On the other hand, the complex of LmFPPS with the inhibitor hydrogen (1-phosphono-2-(1-propyl-1H-imidazol-3-ium-3yl)ethyl)phosphonate (1337), shows clear electron density for the bisphosphonate, a pyrophosphate molecule and three Ca2+ ions without any density for IPP. The crystal of the complex of LmFPPS with the third inhibitor, hydrogen (1-hydroxy-1-phosphono-2-(1-propyl-1H-imidazol-3-ium-3-yl)ethyl)phosphonate (1336), shows clear electron density for the bisphosphonate and three $\mathrm{Ca} 2+$ ions with no electron density for IPP and a pyrophosphate. Comparison of the LmFPPS-bisphosphonate-Ca2+ complex structures show that the bisphosphonates that lack a hydroxyl at the geminal carbon $\mathrm{C} 1$ align well with each other (rmsd $0.25 \AA$ ). The presence of $\mathrm{OH}$ at the $\mathrm{C} 1$ carbon in compound 1336, results in a displacement of the calcium atoms by 0.6-0.8 A. Moreover, Glu-98, a residue of the conserved first aspartate rich motif which is typically a ligand of the divalent cations, is also at hydrogen bonding distance of the 1336-OH group. Though the conformation of the active site residues is very similar in all the structures, Phe-94 in the LmFPPS-1216 structure faces away from the binding pocket of the bisphosphonate aliphatic chain. Interestingly, binding kinetics determined by SPR shows that compound 1216, 3 carbons longer than 1337, has a four-fold higher affinity $(\mathrm{KD}=120 \mathrm{nM})$ than the 1337 compound. Detailed analyses of the crystal structures, binding affinities and cellular activity provide insights into bisphosphonate design for effective LmFPPS inhibition.

Acta Cryst. (2020). A76, a182 